\title{
Construyendo puentes entre la política ambiental y la política de tratamiento de aguas en la cuenca Lerma-Chapala
}

\author{
Raúl Pacheco-Vega*
}

\begin{abstract}
Resumen
El desarrollo sostenible de una nación no reconoce divisiones entre la importancia de los factores económicos, ecológicos, sociales, políticos y culturales; sin embargo, una revisión analítica de política hidráulica y ambiental en México hace patente la existencia de un abismo. Existen divergencias entre dos políticas sectoriales que debieran ser, por definición, convergentes. Para demostrar dicho abismo, en este artículo se examina la política hidráulica en materia de aguas residuales desde la teoría neoinstitucional, considerando la cuenca Lerma-Chapala como caso de estudio. Aquí se argumenta que es posible fortalecer la política hidráulica en materia de tratamiento de aguas residuales mediante el diseño de instituciones cooperativas robustas para la gestión integrada de la cuenca Lerma-Chapala. Esto contribuirá (aunque no garantizará) a la construcción de puentes entre dos políticas tradicionalmente desintegradas.
\end{abstract}

Palabras clave: Lerma-Chapala, política hidráulica, política ambiental, tratamiento de aguas.

\begin{abstract}
The sustainable development of a nation does not recognise divisions between the importance of economical, ecological, social, political and cultural factors. However, an analytical review of the water and environmental policies in Mexico does show a gap between them. There are divergences between two sector policies that must be, by definition, convergent. In order to demonstrate the existence of this gap, this article examines the water policy in terms of residual waters from the neo-institutional theory, considering the Lerma-Chapala basin as a case study. In this article we argue that it is possible to strengthen the water policy in terms of residual water treatment through the design of robust cooperative institutions for the integrated management of the Lerma-Chapala basin. This will contribute (although it will not guarantee) the building of bridges between two policies that traditionally have not been integrated.
\end{abstract}

Keywords: Lerma-Chapala, water policy, environmental policy, water treatment.

* Investigador independiente; asociado por proyectos con el Centro de Innovación Aplicada en Tecnologías Competitivas (CIATEC), León México. Correo-e: raul.pachecovega @gmail.com, rpacheco@ciatec.mx. 


\section{Introducción $^{1}$}

Si bien 75\% de la superficie del planeta Tierra es agua, solamente $2 \%$ de ese volumen es aprovechable. Casi $70 \%$ del agua disponible de la Tierra está en glaciares, y cerca de $18 \%$ de la población total mundial no tiene acceso a fuentes seguras de agua potable (UNESCO, 2004). Ante la gran dificultad que representa la gestión del agua de manera integral, considerando factores económicos, sociales, políticos y biofísicos, la tendencia mundial en años recientes ha sido el enfoque del manejo integrado de cuencas hídricas (integrated watershed management). Estudios recientes (Cotler, 2004) examinan la implantación de ese paradigma en México. De la misma forma, el número de trabajos cuyo objeto de estudio es la cuenca Lerma-Chapala, es ya considerable (Boehm-Schoendube et al., 2002; Boehm-Schoendube y Sandoval-Manzo, 1999; Ramos-Osorio y Wester, 2000; Vargas-Velázquez, 2003; Wester et al., 2001). El interés por esta cuenca se ha incrementado de manera dramática en diversos grupos en los ámbitos nacional e internacional. ${ }^{2}$ Sin embargo, gran parte de la literatura especializada tanto en gestión integrada por cuencas como en esta cuenca en específico, se ha enfocado en dos aspectos primordiales: la gestión integrada por cuencas (GIC) como paradigma, y el manejo del recurso hídrico desde la perspectiva de la escasez. Es decir, se sugiere que la GIC debería de implantarse en la cuenca Lerma-Chapala, dada la condición crítica en la cual se encuentra (el bajo nivel del lago de Chapala con frecuencia se menciona como el principal problema). ${ }^{3}$ Por ello, el discurso predominante en el análisis es que el agua se está acabando y se

${ }^{1}$ Algunas ideas presentadas en este artículo fueron discutidas en una ponencia en el III Encuentro de Investigadores sobre la Cuenca Lerma-Chapala-Santiago, en octubre del 2004, en Chapala, Jalisco. Este artículo es una versión considerablemente diferente a la presentada en la ponencia, si bien algunos conceptos permanecen. Agradezco los comentarios de los dos dictaminadores anónimos de Economía, Sociedad y Territorio. Agradezco el financiamiento del Consejo Nacional de Ciencia y Tecnología de México (CONACYT) y del Consejo de Ciencia y Tecnología del Estado de Guanajuato (CONCYTEG), para llevar a cabo la investigación de la cual se derivan los resultados aquí presentados (clave de proyecto FOMIX GTO-04-106); así como el valiosos apoyo de Fernando Basurto y Manuel Velázquez, asistentes de investigación.

${ }^{2}$ Véase, por ejemplo, el volumen editado por Anne Hansen y Markus van Affereden (2001), así como los libros compilados por Brigitte Boehm-Schoendube y colaboradores (1999 y 2002).

${ }^{3}$ Es interesante, sin embargo, ver que a pesar de que el discurso predominante es la escasez del líquido y las batallas predominantes entre los cinco estados por el agua, los trasvases de y al lago de Chapala, es un hecho comúnmente aceptado (y con frecuencia difundido en los medios masivos de comunicación) que la calidad del agua en el río Lerma es deplorable. No obstante, este tema ha sido poco estudiado en la literatura académica, lo cual resulta, por ende, paradójico. 
requieren formas novedosas para atacar ese problema. Sin embargo, tal vez valdría la pena expandir el debate incluyendo aspectos de saneamiento y tratamiento de las aguas residuales. ${ }^{4}$

$\mathrm{Al}$ incluir elementos del estudio de los instrumentos de política ambiental para prevenir la contaminación, en este artículo se busca estrechar el abismo que existe entre la política ambiental y la política hidráulica, en particular en materia de control de la contaminación. Primordialmente, este análisis privilegia la importancia de las instituciones en la formulación e implantación de política pública. Asimismo, se mantiene como premisa principal que la formación de instituciones robustas (un prerrequisito en la ejecución exitosa de instrumentos de política ambiental) puede ser una herramienta para fortalecer la política hidráulica en México e interconectarla con la política ambiental. Este trabajo, sin embargo, se circunscribe única y específicamente al rubro de control de la contaminación en aguas residuales.

\section{Construyendo puentes entre políticas sectoriales: ambiente, agua y agua residual reexaminados}

La política del agua en México es compleja y multifacética. Una revisión profunda de la literatura (Barkin, 2003 y 2001; CastelánCrespo, 2000a; Tortajada, 2001; Vargas-Velázquez, 2003) indica dos puntos en los cuales hay separación. En primer lugar, existe una desarticulación entre la política ambiental y la política hidráulica (Caire, 2004; Pacheco-Vega, 2005; Tortajada, 2002). Los aspectos relacionados con la sostenibilidad y la viabilidad de conservación del agua no han sido analizados adecuadamente desde una perspectiva de política pública. En segundo lugar, aun dentro de la política hidráulica se ha prestado poca atención al aspecto del conflicto por el uso y disposición final de las aguas residuales. En el caso específico de la cuenca Lerma-Chapala, una revisión de la literatura disponible sobre el tema indica un vacío en los aspectos relacionados con la política de saneamiento.

Un trabajo que se aproxima al estudio de las aguas residuales y la necesidad de establecer políticas y guías de acción para su tratamiento, es el que ofrece Cecilia Tortajada, quien indica que el monto total anual de aguas residuales generadas en el país se calcula en $7.3 \mathrm{~km}^{3}\left(231 \mathrm{~m}^{3} / \mathrm{s}\right)$, de los cuales solamente $5.5 \mathrm{~km}^{3}$ llegan

\footnotetext{
${ }^{4}$ Un trabajo muy reciente, aún no publicado, en el que colaboran varios investigadores del Instituto Mexicano de Tecnología del Agua, como Boris Marañón, Sergio Vargas y Roberto Romero, entre otros, es una contribución al análisis de este tipo de arreglos institucionales.
} 
a sistemas de alcantarillado (Tortajada, 2002). La autora enfatiza que se debe reforzar la coordinación vertical y horizontal y esclarecer la división de responsabilidades entre las instituciones. Desgraciadamente, éste todavía no es el caso en México. El trabajo de Patricia Ávila-García es pionero, ya que ella se enfoca en los conflictos por el deterioro ambiental causado por la mala calidad del agua en el lago de Cuitzeo (Ávila-García 2002). Muchos otros trabajos han postulado la necesidad de mejorar la calidad del agua en México (Ávila-García, 1998; Tortajada, 2002).

En cuanto al caso de estudio propuesto en este trabajo, existe ya una gran cantidad de datos tanto hidrológicos y geológicos como de investigación de carácter social en relación con la cuenca Lerma-Chapala (Boehm-Schoendube et al., 2002). Sin embargo, a pesar de la abundancia de dichos datos, la fragmentación divisoria entre la política del agua y la política ambiental permanece de manera notoria. Esto no sorprende, ya que la política acuífera en general tiende a enfocarse más en la irrigación y la transferencia a los distritos (Ingram, 1990) y menos en el tratamiento de las aguas residuales que circulan por los cuerpos de agua de la cuenca (Pacheco-Vega, 2005; Peña, 2005). Persiste entonces un abismo entre políticas hidráulicas y políticas ambientales.

¿A qué me refiero con el abismo entre las políticas ambientales e hidráulicas? Hay varias respuestas a esta pregunta. En primer lugar, existe una visible división de responsabilidades en las agencias gubernamentales responsables de los aspectos ambientales en México. Si bien la Secretaría de Medio Ambiente, Recursos Naturales y Pesca (Semarnat) tiene en su organigrama a la Comisión Nacional del Agua (CNA) como un organismo "desconcentrado", realmente no hay una integración entre ambas agencias. No hay conexiones directas ni burócratas designados para que colaboren de manera estrecha una organización con la otra. En segundo lugar, los textos de las leyes y reglamentos formulados por la CNA y la Semarnat carecen de convergencia en una serie de aspectos. Para tomar un ejemplo, la responsabilidad de verificar el cumplimiento de la normatividad ambiental fue, hasta el año 2000, de la Procuraduría Federal de Proteccion al Ambiente (Profepa). En teoría, esta institución tendría el respaldo jurídico para verificar que no hubiera contaminación en los ríos. Sin embargo, esta responsabilidad se le asignaba a la CNA. En las leyes y reglamentos municipales, la mayor parte de las responsabilidades del tratamiento de aguas residuales y la calidad del agua son municipales. Las agencias ambientales estatales permanecen 
en un limbo. No obstante, a partir del 2004, y con la reforma a la Ley de Aguas Nacionales (LAN), la Profepa será capaz de tener poderes similares a los de la CNA para el monitoreo. Esto suena, en primera instancia, muy positivo. Pero existe una interrogante: ¿tendrán la Profepa y la CNA la capacidad de monitorear y regular que no existan descargas contaminantes a los ríos? En este momento, la respuesta es no. Todavía estamos a muy poco tiempo de haber puesto en marcha las reformas de la LAN como para analizar si se ha dado un cambio. En tercer lugar, existe un abismo, porque si bien la Semarnat es responsable de la preservación de los recursos naturales, el agua nunca se ve incluida dentro de los recursos naturales como los percibe la agencia gubernamental. Si ése fuera el caso, habría una liga directa entre las subsecretarías encargadas de la gestión de los recursos y sus homólogos de la CNA. Pero en este momento, no existe.

También es importante reconocer que la minimización y control de la contaminación tienen objetivos diferentes a la preservación y gestión sostenible de los recursos naturales, aun cuando ambas están inextricablemente relacionadas (la búsqueda de un desarrollo sotenible). En materia de minimización de los residuos, el objetivo es controlar y modificar el comportamiento de un grupo de agentes (las empresas) para reducir su patrón de generación de contaminantes (Pacheco y Nemetz, 2001). La meta primaria de los instrumentos de política ambiental para el control de la contaminación es la reducción de emisiones hacia distintos medios (agua, aire, suelo). En este sentido, la óptica es diferente en tanto que es relativamente más simple ajustar los patrones de comportamiento del agente contaminante mediante el establecimiento de normas y límites de descarga a cuerpos receptores, la imposición de impuestos ecológicos, o la participación en acuerdos voluntarios para minimizar las emisiones. El diseño del instrumento de política ambiental tiene un enfoque específico y un agente objetivo determinado.

En cambio, cuando se habla de política hidráulica, dicha noción abarca una gran variedad de asuntos: la distribución equitativa del agua, los derechos de propiedad y usufructo de los pozos, los destinos finales del agua residual una vez que es utilizada, el uso agrícola de las aguas residuales, la construcción y puesta en operación de plantas de tratamiento, los sistemas de gestión del agua subterránea, en fin, un sinnúmero de áreas. No es tan 'sencillo' como determinar el agente objetivo y establecer un instrumento de política ambiental que le obligue a modificar su con- 
ducta contaminante. ${ }^{5}$ Por el contrario, hay tantos actores con tan diferentes objetivos, preferencias, intereses, ideas, que resulta bastante más complejo entender la política hidráulica que la política ambiental de control de contaminación.

La primera pregunta que surge es: ¿cuál es, exactamente, el objetivo de la política hidráulica? De la miríada de objetivos que se citan en los documentos oficiales, la escasez de agua y la distribución equitativa del recurso entre los usuarios me parece que son los más citados. Sin embargo, existen muchos otras metas, programas y actividades relacionadas con el manejo del agua que merecen nuestra atención. El problema es que el objeto de nuestro estudio resulta mucho más complejo que las herramientas analíticas que usamos para comprenderlo. Como se acostumbra decir en lenguaje coloquial, "nos queda grande el zapato".

Por ejemplo, se encuentra el caso de la política hidráulica de Guanajuato: si bien el Plan Estatal Hidráulico 2000-2006 de la Comisión Estatal del Agua de Guanajuato (CEAG) dice que el objetivo del Sistema Estatal de Planeación Hidráulica es "establecer las condiciones para el análisis, discusión, formulación y presentación de las acciones para el manejo integral sustentable del agua en el estado" (p. 6), los términos empleados podrían ser más precisos. Resulta importante delimitar a qué se refiere el manejo integral, cómo se define el término sustentable, etc. En materia de política hidráulica, el manejo integral está asociado con la implantación de gestión integrada por cuencas hidrológicas (Cotler, 2004; Ramos-Osorio y Wester, 2000; Vargas-Velázquez, 2003). Pareciera que éste fuera solamente un problema de semántica. Pero, la realidad es que una de las dificultades para comprender los problemas ambientales radica en la imprecisión de los términos utilizados para describirlos. Por ejemplo, el vocablo “desarrollo sustentable” tiene ya más de 70 acepciones diferentes. Sin embargo, la versión originada en el reporte Brundtland parece ser la más socorrida, ya que incorpora la dimensión social, ambiental y económica, así como los aspectos de equidad intergeneracional (Pacheco-Vega et al., 2001). Sin embargo, no queda muy claro cómo se puede a llevar a cabo un manejo integral sostenible del agua. Este grado de 'sostenibilidad del recurso hídrico' se pregona como resultado de la aplicación del paradigma de gestión por cuencas. Tres importantes

${ }^{5}$ Esto no quiere decir que la política de control de contaminación sea más sencilla, sino que tiene un ámbito de interacción y de conceptuación un poco más reducido y menos problemático que la política hidráulica. 
interrogantes referentes a la gestión integrada del recurso hídrico tienen conexión directa con este paradigma.

La primera tiene que ver con el ámbito y la escala de la unidad de análisis. En el caso de la nueva política hidráulica mexicana, se acepta la gestión por cuencas como paradigma o modelo a seguir, pero entonces es posible preguntarse: ¿̇cuál es la mejor forma de organizar las instituciones dentro de una cuenca para la adecuada gestión del agua dentro de la cuenca hidrológica? Como bien argumenta Axel Dourojeanni (2004), sabemos mucho sobre manejo de cuencas, pero todavía no lo hemos puesto en marcha. Una política hidráulica que admite el manejo integrado de cuencas en forma normativa (como el 'deber ser') no responde a esta interrogante. En la opinión del autor de este artículo, no existen todavía las condiciones en México para implantar el manejo integrado de cuencas, si bien hay una serie de esfuerzos tanto por parte de la CNA como por parte de la academia enfocada al estudio de las cuencas hídricas.

La segunda pregunta se focaliza en la delineación de las modalidades de integración. En el momento en que hablamos de manejo integrado, ¿quiénes se convierten en los representantes interesados (stakeholders) que deberán tomar decisiones para beneficio (o perjuicio) de todos los habitantes que se encuentran dentro de la cuenca? (Wester et al., 2003). Éste es un problema de representación de los interesados que no es de tan fácil solución y, por ende, se convierte en nuestra segunda interrogante. Además, es muy importante reconocer la heterogeneidad de los diferentes estados en la cuenca. Algunos estados tienen un Índice de Desarrollo $\mathrm{Hu}-$ mano (IDH) muy alto, como el Estado de Mexico y Jalisco, y existen otros cuyos IDH son menores, como es el caso de Michoacán. El gran problema es que todavía no se llega al grado de madurez analítica necesaria para reconocer las implicaciones de las poblaciones heterogéneas (Merino-Pérez, 2004). No todos podemos adoptar de la misma manera la gestión integrada por cuencas, simplemente porque no todos tenemos las mismas capacidades.

La tercera pregunta se enfoca en la concurrencia de competencias. ¿A quién le compete qué actividad en materia de agua? En materia de gestión integrada de agua por cuencas, se establece teóricamente que la interacción entre los diferentes órdenes de gobierno (municipal, estatal y federal) deberá coordinarse junto con todos los actores adicionales al gobierno (organizaciones nogubernamentales, académicos, representantes de la sociedad civil). Sin embargo, el gran problema es que ni la política hidráuli- 
ca ni ambiental, y mucho menos la política de salud pública, están diseñadas para fortalecer dicha coordinación interjurisdiccional. La tercera interrogante entonces es cómo fortalecer esta coordinación en la República Mexicana, donde todavía es muy incipiente el federalismo.

\section{2. ¿Cómo puede ayudar la teoría neoinstitucional a estrechar los abismos entre la política ambiental y la política hidráulica?}

Para ayudar a estrechar los vínculos entre estas dos ramas de políticas públicas, en este trabajo se hace uso de un marco analítico propuesto en la obra de George Hoberg (2002): el modelo del régimen de política pública (policy regime). En este modelo, Hoberg y sus coautores establecen que para explicar un cambio de política pública, es necesario analizar la influencia de tres variables que forman el régimen: las ideas, los intereses y las instituciones $\left(\mathrm{I}^{3}\right)$. En toda decisión de política, estas tres variables primordiales determinan el resultado de la implantación de dicha política. Las ideas (los valores y nociones predeterminadas que los actores tienen), los intereses (desde el punto de vista racional, las preferencias de los individuos en términos de su interés egoísta) ${ }^{6}$ y las instituciones (definidas como las reglas formales e informales de las interacciones entre los individuos) conforman un régimen de política.

Cuando se aplica este marco analítico, es posible mostrar que en materia de política hidráulica, la importancia de las instituciones como variable explicativa es mucho más alta que la de los intereses o las ideas. Es mucho más sencillo entender las instituciones y las relaciones o arreglos institucionales tanto formales como informales dentro de una cuenca hidrográfica específica, que tratar de determinar o asignar intereses o valores específicos o incluso explorar las ideas y creencias de un actor o grupo de actores. Es obvio que los intereses de los actores (así como sus creencias o ideas) tienen gran relevancia. Dependiendo de quiénes estén involucrados y qué estrategias utilicen, la política puede tener una dirección diferente. Sin embargo, en el presente estudio se parte de la premisa de que los actores participantes tienen intereses e ideas (valores intrínsecos) contrapuestos. Sin embargo, en la evolución de la política hidráulica en México (particularmente en materia de aguas residuales) se ha prestado la mayor atención a los aspectos de instrumento de política ( $\mathrm{y}$ a un instrumento regu-

"También denominado en la literatura anglosajona "self-interest". 
latorio en específico, la Ley de Aguas Nacionales). Por esta razón (el énfasis en los aspectos de gobierno y organizativos) es apropiado analizar la política hidráulica de tratamiento de aguas residuales mediante la perspectiva neoinstitucionalista (North, 1990a; Ostrom, 1991; Pacheco-Vega, 2005; Wester et al., 2001).

Existe un cuerpo sustancial de evidencia empírica sobre la forma en la que las instituciones internacionales modifican los resultados de la política pública (policy outcomes). Un postulado fundamental es que las instituciones moldean las políticas públicas en todas las etapas del ciclo de política (formación de la agenda, diseño de instrumentos, implantación y evaluación) (Pacheco y Nemetz, 2001). En materia de política hidráulica, también la evidencia indica que las instituciones son clave en el éxito o fracaso de la gestión adecuada del recurso hídrico (Fuchs, 2003; Ostrom, 1990, 1991 y 1999; Ostrom et al., 1994 y 2002; Tortajada, 2002; Vargas-Velázquez, 2003; Wester et al., 2001).

Un problema común cuando se usan teorías relacionadas con las instituciones (neoinstitucionalismo, análisis y desarrollo institucional, por mencionar un par de ejemplos), es que en muchas ocasiones se traslapa el término institución a las organizaciones sin que se definan los límites de una y otra adecuadamente. Por ejemplo, la familia es una institución, ya que está delimitada por un conjunto de reglas y principios de organización de una sociedad en específico. Sin embargo, en ocasiones tendemos a hablar de instituciones para referirnos a organizaciones.

En este trabajo, la conceptuación de las instituciones está íntimamente ligada a la estructura de los derechos de propiedad y la forma en la que las reglas de interacción entre los actores están definidas. Douglass North define las instituciones como "las restricciones humanamente desarrolladas que conforman la interacción humana" (1991, 1990a y 1990b). En opinión de North, las organizaciones son diferentes de las instituciones en que las primeras son respuestas endógenas, optimizadoras de los seres humanos respecto de su ambiente institucional. La visión de North permite entonces establecer dos dispositivos analíticos para el análisis institucional de la política de saneamiento dentro de la cuenca: las instituciones (que incluyen las jurisdicciones y el establecimiento de las competencias individuales de cada uno de los actores involucrados en la administración de los recursos acuáticos) y las organizaciones (que incluyen los mecanismos formales de organización que utilizan los actores involucrados para el manejo apropiado de los proyectos de restauración). Desde 
esta visión, la mayor parte de los análisis de los arreglos institucionales en el manejo integrado por cuencas han tendido más a ser arreglos interorganizacionales que arreglos interinstitucionales (Pacheco-Vega y Basurto, 2005a y 2005b).

\section{Acción colectiva, gestión del agua, instituciones y la "Tragedia de los Comunes"}

La noción de que los recursos no son inagotables ha sido estudiada desde hace muchos años, aun cuando Garret Hardin (1968) todavía conserva el honor de haber llamado la atención a los problemas que la sobreexplotación de recursos genera. Hardin enfatizó la problemática de utilizar los recursos de manera indiscriminada, un fenómeno al que llamó la "Tragedia de los Comunes". Desde 1972, cuando se llevó a cabo la Cumbre sobre Desarrollo y el Ambiente Humano en Estocolmo, algunos países han tratado de encontrar maneras para prevenir el agotamiento de los recursos de uso común.

En años recientes, el trabajo de Elinor Ostrom y sus colaboradores (1999 y 1991; Ostrom et al., 1994 y 2002) ha demostrado que existen formas específicas de acción colectiva en las cuales la gobernabilidad de los recursos de acceso común permite minimizar la probabilidad de su agotamiento. El término "recursos de acceso común" (common pool resources) se usa para denotar los recursos naturales que son utilizados por muchos individuos en común, tales como las pesquerías, los acuíferos y los sistemas de irrigación (Ostrom, 1990). Dichos recursos han sido por mucho tiempo sobreexplotados y usados de forma inadecuada por individuos que actúan por interés propio y egoísta (en la línea de la teoría de elección racional). Entre los logros de Ostrom está la integración de la teoría de la elección racional con la teoría institucionalista para elaborar un marco analítico que denomina análisis y desarrollo institucional (institutional analysis and development). Como ella indica, las soluciones convencionales a esa sobreexplotación típicamente involucran la regulación centralizada por parte del gobierno o la privatización del recurso. Sin embargo, de acuerdo con Ostrom, existe un tercer enfoque hacia la solución del problema de "los comunes": el diseño de instituciones cooperativas durables que se organizan y gobiernan por medio de los mismos usuarios del recurso.

Sin embargo, debemos tomar en cuenta que existen arreglos institucionales formales e informales. Los arreglos instituciona- 
les dentro de la cuenca Lerma-Chapala descritos y analizados por Phillipus Wester y colaboradores (2001, 2003 y 2005) son los arreglos formales, que provienen del diseño institucional realizado por las agencias centrales dedicadas al manejo del agua (en este caso, la CNA). A los Consejos de Cuenca se les considera arreglos institucionales formales, espacios de coordinación y diálogo entre los diferentes interesados en una cuenca. Pero existen otros tipos de arreglos institucionales informales; Arun Agrawal (1993) analiza el caso de Dodopani, en India, mostrando que la forma en la cual el gobierno eligió complementar el suministro de agua en Dodopani desmotivó a los residentes de la villa a colaborar para crear soluciones colectivas, lo cual originó la ruptura de instituciones participativas autóctonas y deterioró el suministro de agua potable para muchos de los residentes. Agrawal (1993) argumenta que los gobiernos frecuentemente diseñan cambios institucionales orientados técnicamente, pero olvidan poner suficiente atención al contexto político e institucional que define la dinámica de la lucha de poder en la escala rural, así como las interacciones y realidades de dicho entorno.

Considerando el agua como un bien común de acceso restringido (precisamente un recurso de acceso común, common pool resource), podemos ver que los modelos dominantes de análisis (la tragedia de los comunes, el dilema de los prisioneros y la lógica de la acción colectiva) (López-Ramírez, 2003) de este tipo de problemáticas son inadecuados en un contexto en el que no siempre aplican. En donde sí aplican es en contextos donde los individuos no tienen confianza unos en otros, ni la capacidad o mecanismos para negociar acuerdos legalmente enlazantes y cuando no invierten en mecanismos de monitoreo y cumplimiento para evitar la sobreexplotación (Ostrom, 1990; Ostrom, et al., 1994). De acuerdo entonces con la discusión anterior, el gran reto de la gestión integrada por cuencas es el diseño de instituciones robustas y cooperativas dentro de la cuenca. En la siguiente sección se discute el caso de la cuenca Lerma-Chapala y cómo se puede aplicar el análisis institucional al estudio de la política de tratamiento de aguas residuales.

\section{Instituciones en la cuenca Lerma-Chapala}

De acuerdo con Wester y colaboradores (2001), las necesidades de utilizar un enfoque integrado hacia la gestión del agua han llegado a su punto crítico, debido a la existencia de problemas de alta contaminación del lago de Chapala y a su creciente agota- 
miento tanto por evapotranspiración como por excesivo consumo del agua. Estos autores indican una excesiva transferencia de agua del sector agrícola hacia los sectores industriales y urbanos sin la debida compensación. ${ }^{7}$ Sin embargo, se considera que la creación de acuerdos interinstitucionales y de coordinación (como el convenio de coordinación de la cuenca Lerma-Chapala, firmado en 1989, y la creación de un Consejo de Cuenca en 1993) contribuirá a la sostenibilidad del manejo del agua.

En la actualidad existen 26 Consejos de Cuenca en México (mapa I). La Región Hidrológica VIII (Lerma-Santiago-Pacífico) cuenta con tres Consejos (15 Lerma-Chapala, 16 Río Santiago y 17 Costa Pacífico Centro). La cuenca Lerma-Chapala abarca territorio de cinco estados de la República (Querétaro, Michoacán, Estado de México, Jalisco y Guanajuato).

De los 26 Consejos, el Consejo de la cuenca Lerma Chapala se considera uno de los más importantes (Muñiz-San-Martín, 2004). Dicha cuenca representa 3\% del territorio nacional, con una extensión de $54,000 \mathrm{~km}^{2}$ distribuidos en diferentes proporciones entre cinco estados (Boehm-Schoendube et al., 2002).

La cuenca Lerma-Chapala se localiza en la parte central del país y es una cuenca a la que se le ha dado una gran importancia en estudios tanto en México como en el extranjero, no sólo por la cantidad de personas que residen en la cuenca y su "área de influencia" sino también por la gran actividad industrial y económica que presentan. ${ }^{8}$ La cuenca Lerma Chapala está definida como el territorio en el que toda el agua superficial escurre directamente hacia el río Lerma o hacia afluentes, río que, a su vez, desemboca en el lago de Chapala. Sin embargo, existen dos subcuencas cerradas en Michoacán: las cuencas de los lagos de Pátzcuaro y de Cuitzeo.

La población en la cuenca representa $10 \%$ de la del país, mientras que la producción económica y agrícola en el territorio de la misma es 35\% del Producto Interno Bruto (РІB) (Castelán-Crespo, 2000b). El río Lerma, principal afluente de la cuenca, tiene una longitud de $750 \mathrm{~km}$. Nace en el Estado de México, en el centro del país, a 3,000 metros sobre el nivel del mar (msnm) y

${ }^{7}$ Cabe hacer notar que el trabajo de Wester et al. (2001) analiza precisamente los arreglos institucionales dentro de la cuenca Lerma-Chapala, si bien no es específico en materia de aguas residuales.

${ }^{8}$ Agradezco la colaboración del personal de la Dirección General de Cuencas Hídricas del INE, en especial a la doctora Helena Cotler, a la maestra Georgina Cairé y al geógrafo Carlos Enríquez, quien muy amablemente me facilitó algunos mapas e información para este artículo. 


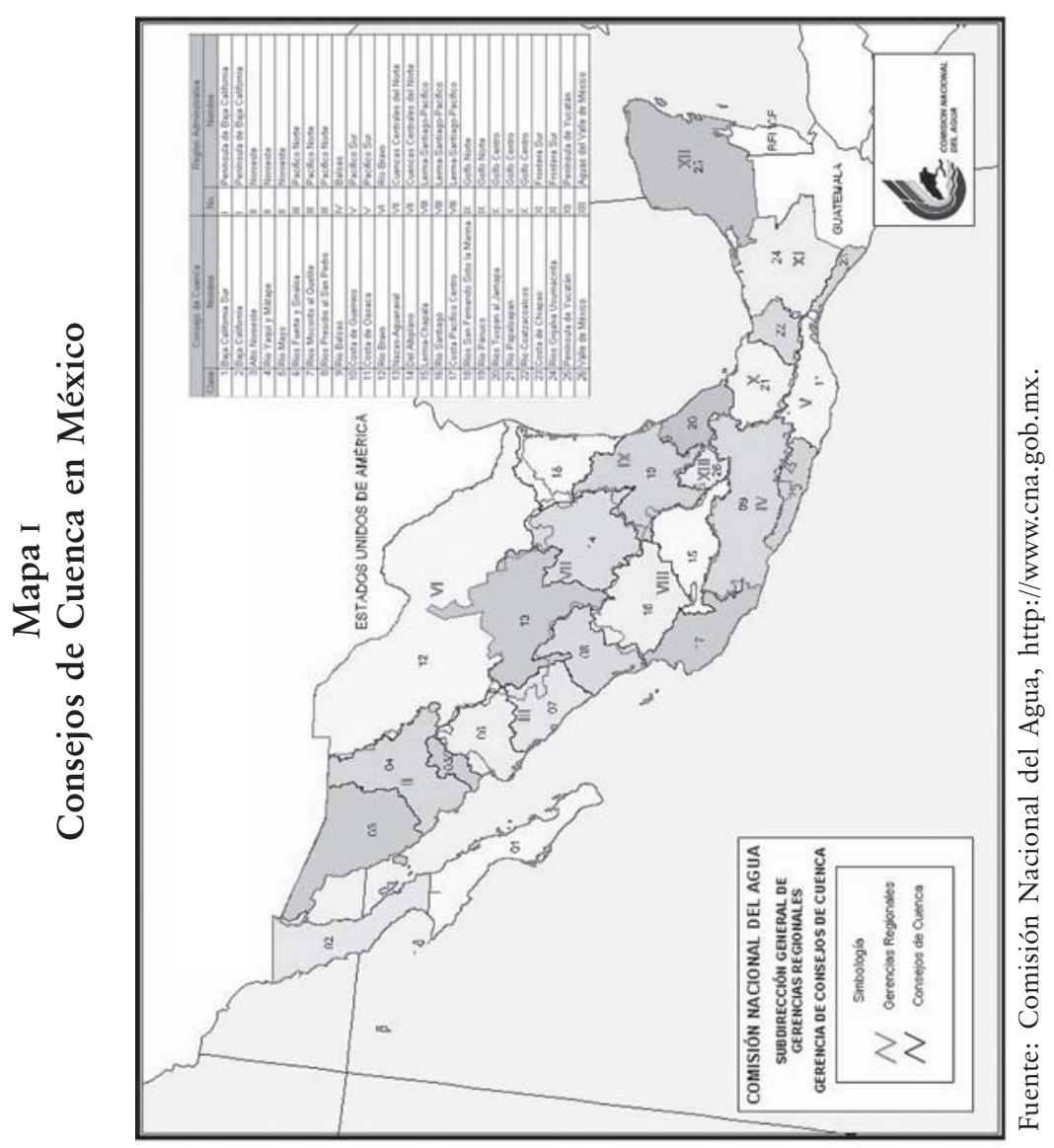




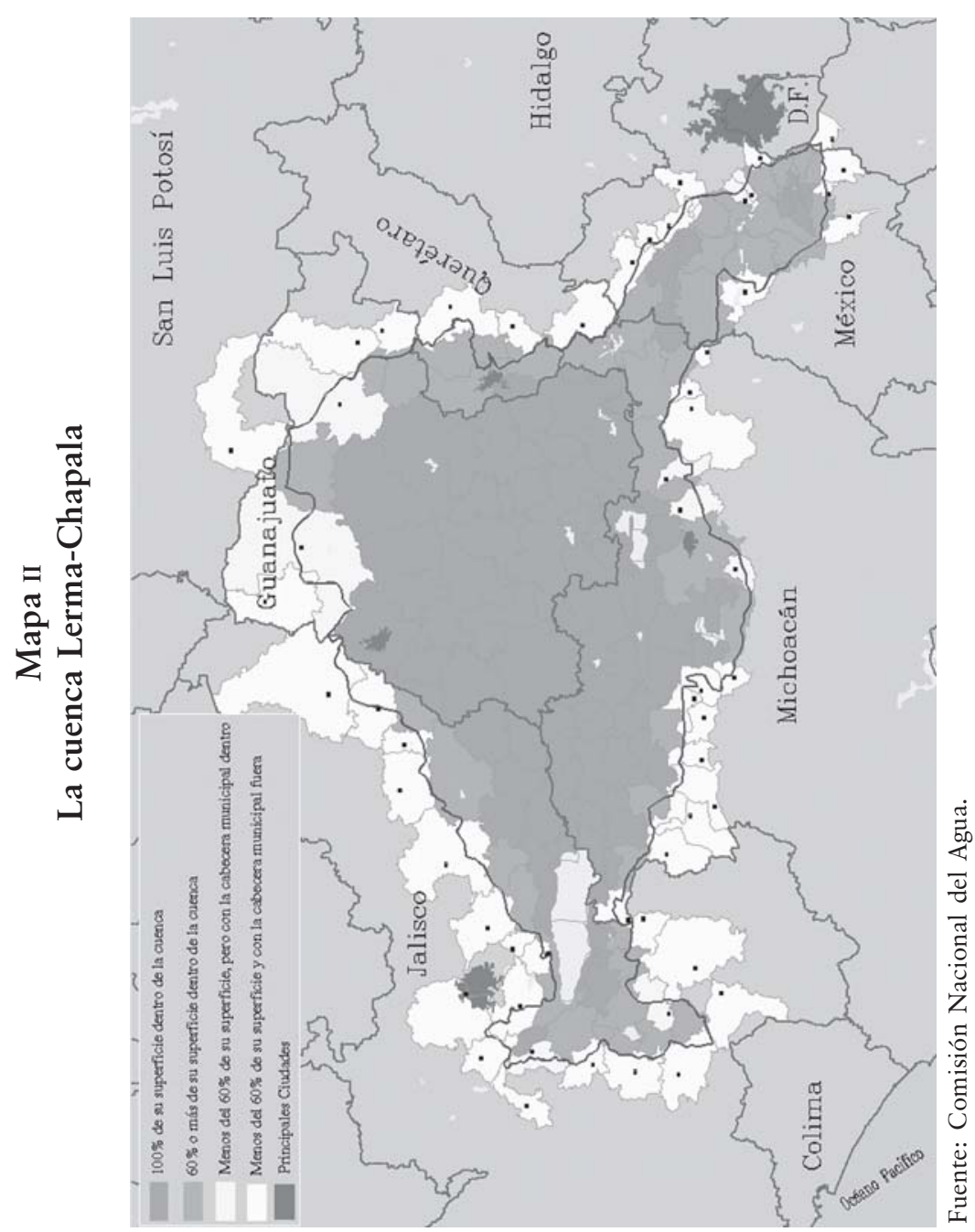


termina en el lago de Chapala a 1,510 msnm, en el occidente del país (Hansen y Van Affereden, 2001).

La historia del Consejo de Cuenca Lerma-Chapala tiene su primer antecedente el 13 de abril de 1989, cuando los gobernadores de los cinco estados que abarcan la cuenca firmaron un acuerdo de coordinación. Ese acuerdo surgió luego de que los gobernadores reconocieron una serie de problemas como contaminación, escasez y sequías, entre otros (Pacheco-Vega, 2005; Peña, 2005; Wester et al., 2001 y 2005). Un nuevo acuerdo, el Convenio de Coordinación y Concertación para la Distribución de las Aguas Superficiales de la Cuenca Lerma-Chapala, se firmó recientemente (2004), especificando, entre otros elementos, las fórmulas para el acuerdo de distribución de aguas superficiales. En el año 2004, a partir de los cambios en la Ley de Aguas Nacionales, se comenzó a dar más importancia al aspecto de las aguas residuales. En el seno del Consejo de Cuenca Lerma-Chapala se formó un Grupo de Trabajo de Saneamiento (que en 20052006 fue presidido por el ingeniero Raúl Almeida Jara, director de Saneamiento de la Comisión Estatal del Agua de Guanajuato, CEAG). Ese grupo indicó que el programa de saneamiento de la cuenca tenía como objetivo construir un cierto número de plantas de tratamiento de aguas residuales en un periodo específico.

Un análisis de contenido de noticias impresas en los medios de comunicación hace patente que la preocupación primordial en el estudio de la cuenca Lerma-Chapala es la preservación, el mantenimiento y la recarga de los cuerpos de agua. ${ }^{9}$ La política de saneamiento del agua residual continúa siendo de poca importancia en la literatura. ¿A quién le compete tratar el agua residual? ¿A los organismos operadores? Si ése es el caso (en la escala de jerarquía gubernamental, correspondiendo al nivel municipal), entonces, ¿quién se ocupa del agua residual generada en las actividades agrícolas o industriales que no se encuentran localizadas en la zona urbana? Los debates en la literatura se centran en la necesidad de mantener los niveles del lago de Chapala, pero poco o nada se indica sobre quiénes son responsables de mantener la calidad del agua en el río Lerma en niveles aceptables. En teoría, el Consejo de Cuenca Lerma-Chapala debería tener responsabilidades y poderes suficientes para poder instrumentar programas de saneamiento.

${ }^{9}$ El Instituto Mexicano de Tecnologia del Agua, el Centro Mexicano del Tercer Mundo para el Manejo del Agua y el CIATEC han sido pioneros en el estudio de aspectos de aguas residuales e instituciones. 
Por otro lado, en el cuadro 1 se muestra que, en el seno del Consejo de Cuenca Lerma-Chapala, la política de saneamiento está establecida en términos del número de plantas de tratamiento de aguas residuales municipales que se construyen en los cinco estados de la cuenca. El total del programa (100 plantas más cinco ampliaciones), sin embargo, no establece objetivos específicos de calidad del agua (por ejemplo, al término del programa el río Lerma llevará agua residual con como máximo $500 \mathrm{mg} / \mathrm{l}$ de demanda bioquímica de oxígeno). Esto es una falla sustancial en el diseño de la política de tratamiento de aguas residuales, ya que el objetivo final (reducir el nivel de contaminación en la cuenca) no se cumple, aun bajo la premisa de la contribución a dicho objetivo.

En virtud de la discusión anterior, sería posible inferir que un Consejo de Cuenca vigoroso y coordinado con los diferentes gobiernos estatales y el gobierno federal (tanto la CNA como la Profepa y la Semarnat) podría fortalecer la política de saneamiento en la cuenca. Esto es, si hubiera reglas de organización e interacción robustas (si existiesen instituciones robustas). Varios autores han mostrado ya que el Consejo de Cuenca LermaChapala no es una institución robusta (Caire, 2004; Muñiz-SanMartín, 2004; Pacheco-Vega y Basurto, 2005a y 2005b; Rodríguez et al., 2003; Wester et al., 2001). El análisis presentado en este trabajo viene a confirmar este hecho.

Otra vertiente de mi argumento es que el principio de gestión del agua basado en la disponibilidad (o escasez) del líquido está

\section{Cuadro 1}

Compromisos contraídos por el Consejo de Cuenca LermaChapala y los estados en materia de plantas de tratamiento de aguas residuales

\begin{tabular}{llrrr}
\hline Fase & $\begin{array}{c}\text { Fecha de } \\
\text { firma }\end{array}$ & $\begin{array}{c}\text { Número total } \\
\text { de plantas }\end{array}$ & $\begin{array}{r}\text { Volumen a } \\
\text { tratar }(l / s)\end{array}$ & $\begin{array}{c}\text { Costo } \$ M \\
\text { (millones de } \\
\text { pesos) }\end{array}$ \\
\hline Primera & Abril 1989 & 48 plantas & $3,700 \mathrm{lps}$ & $\$ 339 \mathrm{M}$ \\
Segunda & $\begin{array}{l}\text { Enero 28, } \\
\text { Total del }\end{array}$ & $\begin{array}{r}52 \\
\text { plantas }+\end{array}$ & $10,835 \mathrm{lps}$ & $\$ 722 \mathrm{M}$ \\
programa & 5 ampliaciones & & \\
\hline
\end{tabular}

Nota: Al término del programa se estaría tratando $85 \%$ del agua residual municipal generada en la cuenca.

Fuente: Wester et al., (2001), con datos de la CNA de 1999. 
ligado directamente a la provisión de agua fresca (sin contaminar). Aun cuando ocasionalmente se menciona como parte de la discusión sobre manejo de agua, se da poca atención tanto a la asignación de responsabilidades como a la búsqueda de mecanismos para minimizar la contaminación de los cuerpos de agua. Aquí es precisamente donde entra la perspectiva de política ambiental de la que hice mención al inicio del trabajo: es imprescindible establecer clara y explícitamente quiénes son los actores objetivo de la política, así como los diferentes mecanismos para el control y modificacion de sus patrones de comportamiento. En el caso del agua residual dentro de una cuenca hidrográfica, la responsabilidad requiere estar más focalizada. No pueden quedarse fuera de los sistemas de tratamiento de aguas residuales aquellas corrientes que sean de jurisdicción federal y que pasen por regiones de jurisdicción municipal. Es aquí donde se hace necesaria la coordinación entre los tres niveles de gobierno, así como las distintas agencias encargadas del monitoreo y vigilancia del cumplimiento de la legislación ambiental. Por ejemplo, en el caso de la ciudad de León, en el estado de Guanajuato, la responsabilidad del tratamiento de aguas residuales es del Sistema de Agua Potable y Alcantarillado de León (SAPAL), y del cumplimiento de las condiciones de descarga es la Gerencia de Fiscalización Ecológica del SAPAL. Sin embargo, a pesar de que hasta el momento no es directamente competencia de la Profepa, sí es importante comprender que todas las emisiones de residuos (ya sean a la atmósfera, o residuos sólidos o a cuerpos acuáticos) son relevantes y deben ser monitoreadas.

La primera brecha entre política ambiental y política hidráulica puede adjudicarse a la identificación de los agentes objetivo de la política (en este caso, hidráulica), aun si esto no garantiza por sí mismo la gestión sostenible del recurso. Suponiendo que pudiéramos controlar el comportamiento de los actores objetivo (los usuarios del agua) para evitar o minimizar el agotamiento de los acuíferos, la sobreexplotación de los cuerpos superficiales de agua, el desecamiento de los ríos, etc., aun así tendríamos que intentar controlar otros elementos del ecosistema cuya manipulación escapa a nuestras manos: esto es, los ciclos hidrológicos. Aún no se ha descubierto la forma de manipular la frecuencia de las lluvias, la cantidad de precipitación, las velocidades de recarga de los acuíferos, la velocidad de migración por filtración del agua hacia los mantos freáticos, etc. Por tanto, podremos intentar controlar los elementos antropogénicos y algunos de los 
elementos del ecosistema circundante, pero no el ciclo hidrológico ni los elementos climáticos. ${ }^{10}$

La segunda brecha que existe entre política ambiental y política hidráulica es en materia de monitoreo y cumplimiento de la normativa ambiental. Ese abismo existe por dos causas primordiales:

- La falta de capacidad institucional en la Profepa para poder monitorear todas las industrias que tienen emisiones contaminantes en diferentes medios (agua, aire, suelo). Hay muy pocos inspectores para realizar visitas de inspección en todas las empresas e industrias que descargan aguas residuales o tienen emisiones atmosféricas o basura tóxica. Por ende, es sumamente difícil tener un programa de monitoreo riguroso y de cumplimiento de la legislación ambiental.

- La falta de coordinación intersectorial entre las diferentes agencias gubernamentales cuyos intereses y responsabilidades tocan algún aspecto relacionado con salud humana, calidad ambiental, desarrollo económico, los cuales son todos elementos de un verdadero desarrollo sostenible. Esa falta de coordinación se traduce en que se tienen diferentes agencias en los tres niveles de gobierno que se abocan a la protección del ambiente y al manejo de agua (residual en algunos casos, potable en otros) y que realizan esfuerzos aislados y sin coordinación, lo cual conlleva el debilitamiento de la sostenibilidad en materia hidráulica en México.

En la siguiente sección se describen las reformas a la Ley de Aguas Nacionales y su efecto sobre la política del tratamiento de aguas residuales. En particular, la discusión se enlaza con la institución que (en teoría) debería promover el saneamiento en la cuenca, el Consejo de Cuenca.

\section{La reforma a la Ley de Aguas Nacionales y los arreglos interorganizacionales}

La reforma de la Ley de Aguas Nacionales que entró en vigor el 30 de abril del 2004 se sustenta en tres principios primordiales: ${ }^{11}$

\footnotetext{
${ }^{10}$ Es cierto que algunos elementos del ciclo pueden ser manipulados, pero la mayor parte del mismo es realmente inamovible.

11 http://www.cna.gob.mx/publica/doctos/publicaciones/D300404CJJ-LAN.pdf.
} 
- Lograr que la administración de las aguas nacionales se realice por cuenca hidrológica, al ser ésta el lugar en el que deben tomarse las decisiones en cuanto a su gestión.

- Lograr la participación de los órganos de gobierno, de los usuarios y de la sociedad en la toma de decisiones.

- Lograr el fortalecimiento de las funciones de las autoridades del agua.

En este nuevo modelo se fomenta la gestión integrada por cuentas y se crean organismos (Organismos de Cuenca) que tienen como objetivo fortalecer la participación social en la gestión del agua en la cuenca. Sin embargo, a pesar que en teoría la CNA está fomentando la gestión integrada por cuenca, su estructura organizacional y el marco jurídico mexicano no permiten realmente implantar ese tipo de gestión. En las figuras I, II y III se muestra la estructura organizacional de la CNA y de varias de sus dependencias que tienen que ver con la operación del Consejo de Cuenca Lerma-Chapala.

En teoría, debería existir una Gerencia de Cuencas o Dirección de Cuencas global en la CNA. Sin embargo, en la figura I podemos ver que la jurisdicción de los aspectos de manejo integrado por cuencas se encuentra en la Subdirección General de Programas Rurales y Participación Social. También es importante notar que las gerencias regionales pertenecen a una Subdirección General de Gerencias Regionales (figuras II y III), que no parece estar (al menos desde el punto de vista organizacional) coordinada con la Gerencia de Cuencas. Es decir, existen dos ramas generales de la CNA que tienen objetivos que deberán estar integrados. El reto es que, en la operación, realmente funcionen de esta manera.

La Gerencia de Consejos de Cuencas debería trabajar en forma coordinada con cada una de las gerencias regionales. En el caso que nos ocupa, la Gerencia de Consejos de Cuencas deberá coordinarse con la Gerencia Regional Lerma-Santiago. Asimismo, se debería poner énfasis en el trabajo coordinado con las comisiones estatales del agua.

También es curioso observar que dos estados que tradicionalmente no están considerados en la cuenca Lerma-Chapala se incluyan en la Gerencia Regional Lerma-Santiago (Nayarit y Colima). Esta inclusión parece provenir del hecho de considerar el río Santiago como parte del sistema Lerma-Chapala-Santiago. Sin embargo, los retos que aquejan a la cuenca Lerma-Chapala no son exactamente los mismos que los del sistema Lerma-Chapala-San- 


\section{Figura I}

\section{Estructura organizacional de la CNA}

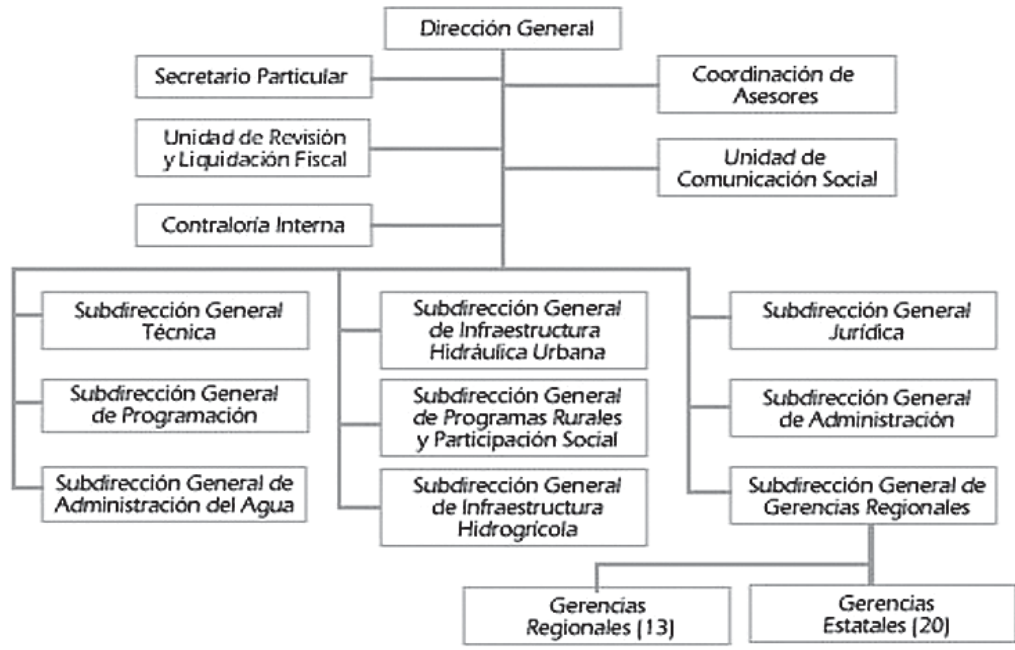

Fuente: http://www.cna.gob.mx.

Figura II

Estructura organizacional de la Subdirección General de Programas Rurales y Participación Social de la CNA

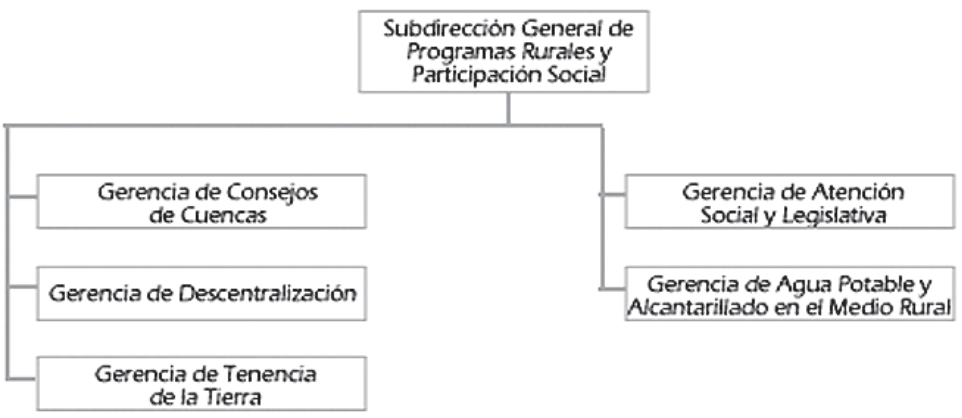

Fuente: http://www.cna.gob.mx.

tiago (ni tampoco se da la misma relevancia a Colima y Nayarit que a Michoacán, Jalisco, Estado de México, Querétaro y Guanajuato). Finalmente, es posible notar que no hay ligas directas entre las gerencias regionales de la CNA y los departamentos encargados de la calidad del agua residual, ni con la Profepa. Por ende, esto demuestra de nuevo que la política federal de tratamiento de aguas residuales y saneamiento continúa desligada de la política ambiental y de sus mecanismos de monitoreo y sanción. 
Figura III

Estructura organizacional de la Gerencia Regional LermaSantiago, perteneciente a la Subdirección General de Gerencias Regionales de la CNA

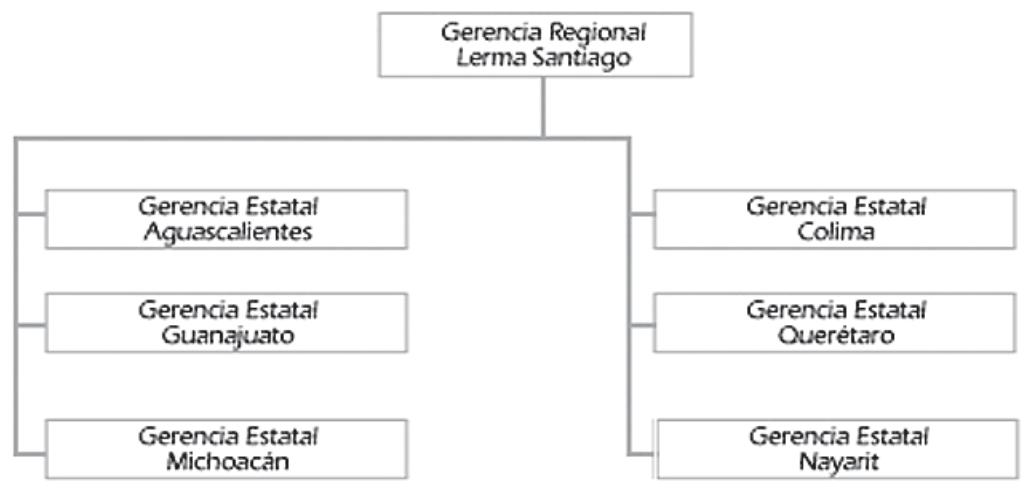

Fuente: http://www.cna.gob.mx.

Es importante notar que éstas reformas están presentes en el papel, pero su ejecución no es tan sencilla como pareciera a primera vista, por las razones que ya muchos otros autores han enfatizado y que se retoman en este trabajo: la administración del recurso agua es mucho más compleja de lo que se ha delineado en las leyes referentes a la gestión del recurso hídrico, sin importar las reformas que se hayan promulgado.

Deberá observarse que el arreglo organizacional (según lo presenta la CNA) del manejo integrado por cuencas involucra una política hídrica obligatoria, lo cual lleva a recordar los instrumentos de política ambiental de tipo comando-control (Pacheco y Nemetz, 2001; Vega-López y Pacheco-Vega, 2000). La pregunta obligada es: ¿cómo pretendemos que la sociedad se involucre en el manejo y gestión del agua cuando los instrumentos de política hídrica resultan ser 'obligatorios' y, por ende, coercitivos? ¿En dónde quedan los espacios de diálogo y concertación para una autogestión del recurso hidráulico? Éstas son algunas interrogantes que podrán dilucidarse hasta cierto tiempo después de la implantación de las reformas a la Ley de Aguas Nacionales (posiblemente en el 2007 o 2008). En este momento no es posible hacer este tipo de análisis. 


\section{Discusión y reflexiones finales}

De las reflexiones presentadas en páginas anteriores, se desprende que la perspectiva de la literatura sobre instituciones debería estar mucho menos enfocada en el estudio de los arreglos de coordinación interorganizacional y mucho más en los conceptos de institución como una serie de reglas que regulan el comportamiento de los agentes objetivo. En ese sentido, en este artículo se sigue la tesis de Ostrom que dice que, en su esfuerzo por construir entidades autogobernadas, "los individuos que se involucran seriamente en esfuerzos para construir relaciones sociales mutuamente productivas son capaces de diseñar formas ingeniosas de relacionarse constructivamente unos con otros, pero dichos individuos son incapaces de construir sistemas sociales enteros desde el principio". ${ }^{12}$

La perspectiva de Ostrom y sus colaboradores permite visualizar algunas reglas para el diseño de los mecanismos de coordinación para la gestión del recurso hídrico. Ostrom enfatiza la necesidad de entender la emergencia, adaptación, diseño y efectos de los arreglos institucionales (formales e informales) para entender una gran diversidad de reglas que existen en una diversidad de entornos físicos y sociales. Más allá de los arreglos interorganizacionales, el interés primordial está en los mecanismos de gobierno del recurso hídrico (específicamente en relación con el manejo del agua residual) dentro de los contextos de la cuenca.

Algunos elementos adicionales que valdrá la pena examinar e interrogar a futuro incluyen: ${ }^{13}$

- El estudio de las reglas de acceso al recurso hídrico, específicamente en la modalidad de agua residual (¿a quién le compete acceder el recurso? Si un usuario tiene acceso al agua, también se hace responsable de su saneamiento como lo pide el principio de "el que contamina, paga"?).

- La definición de los niveles de corresponsabilidad del uso, usufructo y restauración del agua (¿quién se debe hacer responsable de las aguas residuales? ¿Es posible utilizar una forma de gobernabilidad del agua de tipo policéntrico, como plantea Ostrom?).

${ }^{12}$ Estos comentarios surgieron de una reunión personal del autor con Elinor Ostrom y Vincent Ostrom en el verano del 2003 durante su estancia como profesores visitantes.

${ }^{13}$ Este trabajo ya se encuentra avanzado, y un par de artículos escritos sobre el tema por el mismo autor se encuentran bajo evaluación en revistas arbitradas. 
- El surgimiento y conformación de los arreglos institucionales formales e informales (¿qué tan íntimamente ligados se encuentran los arreglos interorganizacionales con los arreglos institucionales formales prediseñados por el gobierno? ¿Cuál es la eficiencia relativa de dichos arreglos?).

- El concepto de escala (¿están dadas las condiciones en México para una gestión integrada de los recursos acuáticos? ¿Qué acciones podemos emprender para simplificar y operacionalizar el manejo sostenible del agua?).

- Los esquemas de solución de los problemas de coordinación entre los diferentes niveles de gobierno, las agencias gubernamentales ambientales y los actores privados, tanto urbanos como industriales y rurales.

- La estructura interna de la cuenca en términos de la complejidad del sistema y el anidamiento de las subestructuras complejas (siguiendo la línea de pensamiento de la teoría de sistemas complejos adaptivos).

A lo largo del artículo, y por medio del examen analítico de la literatura sobre política del agua visto desde la perspectiva de política ambiental, se dilucidan siete lecciones/contribuciones que a continuación se detallan para su discusión:

1) Las instituciones importan mucho en el manejo sostenible del agua. Por ello, una visión institucionalista ofrece gran poder analítico y explicativo, pero no por eso constituye el único elemento para explicar la eficiencia o ineficiencia de la política hidráulica. Se requiere una mayor exploración del asunto.

2) El concepto de arreglos institucionales no significa necesariamente arreglos interorganizacionales, y, por ende, es necesario estudiar la conformación de los arreglos institucionales formales (diseñados por el gobierno) y los informales (establecidos por los usuarios e interesados en la conservación del recurso hídrico).

3) Se ha desatendido el aspecto del manejo del agua residual dentro de las cuencas, prestándose mayor atención a la distribución, recuperación de los cuerpos acuíferos, uso agrícola y transferencia de los distritos de irrigación a los usuarios. Se requiere ahondar en esta área a la brevedad.

4) El manejo del agua es muy complejo y requiere de un análisis multidisciplinario y de tipo multicriterio, así como de una gran variedad de herramientas teóricas y analíticas. 
5) El Consejo de Cuenca Lerma-Chapala como tal no es todavía una institución robusta. Por ende, requiere de fortalecimiento e incluso se deberá considerar la posibilidad del rediseño del Consejo como tal.

6) La brecha entre política ambiental y política hidráulica puede estrecharse mediante una reflexión sobre los agentes objetivo y los instrumentos de política que se utilizan en un campo (ambiental) para su aplicación en el otro (agua).

7) No debemos olvidar que el agua es finalmente un recurso, $\mathrm{y}$, por ello, las agencias encargadas del desarrollo sostenible deberían trabajar en forma integrada y establecer políticas intersectoriales y mecanismos de coordinación suficientemente sofisticados como para una instrumentación adecuada.

Se espera que la contribución mayor de este artículo sea retomar el debate sobre la necesidad y los mecanismos y tecnologías para reducir los volúmenes de efluentes contaminados que se descargan en los cuerpos receptores, ya que éste es uno de los prerrequisitos para un ecosistema sano y sostenible.

\section{Bibliografía}

Agrawal, Arun (1993), "Removing Ropes, Attaching Strings: Institutional Arrangements to Provide Water", manuscrito no publicado.

Ávila-García, Patricia (2002), “Escasez y contaminación del agua en la cuenca del lago de Cuitzeo: el caso de Morelia y su entorno rural", en Brigitte Boehm-Schoendube et al., (eds.), Los estudios del agua en la cuenca Lerma-ChapalaSantiago, El Colegio de Michoacán-Universidad de Guadalajara, Zamora, Michoacán.

Ávila-García Patricia (1998), Social Conflicts, Water Scarcity and Pollution in Mexico, Congres International de Kaslik-Liban, Kaslik, Liban.

Barkin, David (2003), “Innovaciones mexicanas en el manejo del agua: respuestas locales frente a la globalización centralizadora”, Reunión Internacional de la Asociación de Estudios Latinoamericanos, Dallas, Texas. 
Barkin, David (ed.) (2001), Innovaciones mexicanas en el manejo del agua, Universidad Autónoma Metropolitana, Xochimilco-стмma-Centro de Ecología y Desarrollo, México.

Boehm-Schoendube, Brigitte, Juan Manuel Durán-Juárez, Martín Sánchez-Rodríguez y Alicia Torres-Rodríguez (eds.) (2002), Los estudios del agua en la cuenca Lerma-Chapala-Santiago, El Colegio de Michoacán-Universidad de Guadalajara, Zamora, Michoacán.

Boehm-Schoendube, Brigitte y Margarita Sandoval-Manzo (1999), "La sed saciada de la Ciudad de México y la nueva cuenca Lerma-Chapala-Santiago: un ensayo metodológico de lectura cartográfica", Relaciones, xx (80): 15-68.

Caire, Georgina (2004), "Retos para la gestión ambiental de la cuenca Lerma-Chapala. Obstáculos institucionales para la introducción del manejo integral de cuencas", en Helena Cotler (ed.), El manejo integral de cuencas en México. Estudios y reflexiones para orientar la política ambiental, Instituto Nacional de Ecología, México.

Castelán-Crespo, J. Enrique (2000a), Análisis y perspectiva del recurso hídrico en México, CTMMA-CIIEMAD IPN, México.

Castelán-Crespo, J. Enrique (2000b), “Los Consejos de Cuenca en México", en Phillipus Wester et al., (eds.), Asignación, productividad y manejo de recursos hídricos en Cuencas, IWMI, México.

Cotler, Helena (ed.) (2004), El manejo integral de cuencas en México. Estudios y reflexiones para orientar la política ambiental, Instituto Nacional de Ecología, México.

Dourojeanni, Axel (2004), "Si sabemos tanto sobre qué hacer en materia de gestión integrada del agua y cuencas ¿Por qué no lo podemos hacer?", en Helena Cotler (ed.), El manejo integral de cuencas en México. Estudios y reflexiones para orientar la política ambiental, Instituto Nacional de Ecología, México. 
Fuchs, Doris (2003), An Institutional Basis for Environmental Stewardship, Kluwer Academic Publishers, Dordrecht.

Hansen, Anne y Markus van Affereden (eds.) (2001), The LermaChapala Watershed: Evaluation and Management, Nueva York, Kluwer Academic Publishers-Plenum Publishers.

Hardin, Garret (1968), “The Tragedy of the Commons”, Science, 162 (1): 1243-1248.

Hoberg, George (2002), "Policy Cycles and Policy Regimes: A Framework For Studying Policy Change", en Benjamin Cashore, et al. (eds.), In Search of Sustainability: British Columbia Forest Policy in the 1990s, UBC Press Vancouver, Canadá.

Ingram, Helen M. (1990), Water Politics: Continuity and Change, University of New Mexico Press, Albuquerque, N. M.

López-Ramírez, Eduardo (2003), "La costumbre contra la ley: pesca artesanal y gestión ambiental en el lago de Pátzcuaro, Michoacán”, Regiones, 0 (12): 149-166.

Merino-Pérez, Leticia (2004), Conservación o deterioro: el impacto de las políticas públicas en las instituciones comunitarias y en los usos de los bosques en México, Secretaría de Medio Ambiente y Recursos Naturales-Instituto Nacional de Ecología-Consejo Civil Mexicano para la Silvicultura Sostenible, A.C., México.

Muñiz-San-Martín, Sigrid (2004), "Un estudio del proceso de organización del Consejo de Cuenca: el caso de la cuenca Lerma-Chapala en México", en Brigitte Boehm-Schoendube (ed.), Memorias del III Encuentro de Investigadores de la Cuenca Lerma-Chapala-Santiago, El Colegio de Michoacán-Universidad de Guadalajara, Chapala, Jalisco.

North, Douglass (1990a), Institutions, Institutional Change and Economic Performance, Cambridge University Press, Cambridge, Reino Unido.

North, Douglass (1990b), "Institutions, Transaction Costs and Economic Growth”, Economic Inquiry, 25 (3): 419-428. 
North, Douglass (1991), "Institutions”, Journal of Economic Perspectives, 5 (1): 97-112.

Ostrom, Elinor (1990), Governing the Commons. The Evolution of Institutions for Collective Action, Cambridge University Press, Cambridge.

Ostrom, Elinor (1991), “Institutional Analysis, Design Principles and Threats to Sustainable Community Governance and Management of Commons", en E. Berge y N. C. Stenseth (eds.), Law and the Governance of Renewable Resources: Studies from Northen Europe and Africa, ICs Press, Oakland, CA.

Ostrom, Elinor (1999), “The Institutional Analysis and Development Approach", en S. Langill (ed.), Institutional Analysis: Readings and Resources for Researchers, International Development Research Centre, Ottawa.

Ostrom, Elinor, Roy Gardner y James Walker (1994), Rules, Games, \& Common-Pool Resources, The University of Michigan Press, Ann Arbor, MI.

Ostrom, Elinor, Clark Gibson, Sujai Shivakumar y Krister Andersson (2002), Aid, Incentives and Sustainability. An Institutional Analysis of Development Cooperation, Workshop in Political Theory and Policy Analysis, Indiana University, Bloomington, IN.

Pacheco, Raúl y Peter N. Nemetz (2001), Business-Not-As-Usual: Alternative Policy Instruments for Environmental Management, 5th IRE Annual Workshop: Addressing the Knowledge Crisis in Water and Energy: Linking Local and Global Communities, Institute for Resources and Environment, UBC, Vancouver, B.C.

Pacheco-Vega, Raúl (2005), Applying the Institutional Analysis and Development Framework to Wastewater Management Policy in the Lerma-Chapala River Basin, International Workshop Water and Ecosystems: Water Resources Management in Diverse Ecosystems and Providing for $\mathrm{Hu}-$ man Needs, Hamilton, ON, Canadá. 
Pacheco-Vega, Raúl y Fernando Basurto (2005a), "Análisis institucional de la política de saneamiento en la cuenca Lerma-Chapala", II Congreso Iberoamericano sobre Desarrollo y Medio Ambiente, Puebla, México.

Pacheco-Vega, Raúl y Fernando Basurto (2005b), "Reglas Formales e Informales en el Consejo de Cuenca Lerma-Chapala”, Taller Nacional Preparatorio hacia el Foro Mundial del Agua, Instituto Mexicano de Tecnología del Agua, Jiutepec, Morelos, Mexico.

Pacheco-Vega, Raúl, María del Carmen Carmona-Lara y Obdulia Vega-López (2001), "The Challenge of Sustainable Development in Mexico", en Peter N. Nemetz, (ed.), Bringing Business on Board: Sustainable Development and the $B$-School Curriculum, JBA Press Vancouver, B.C.

Peña, Francisco (2005), "El saneamiento de la cuenca LermaChapala. ¿Nudos tecnológicos o baches financieros?”, en Brigitte Boehm-Schoendube et al., (ed.), Los estudios del agua en la cuenca Lerma-Chapala-Santiago, El Colegio de Michoacán-Universidad de Guadalajara, Zamora, Michoacán.

Ramos-Osorio, Roberto y Phillipus Wester (2000), El manejo del agua en la cuenca Lerma-Chapala, México, IWMI Contract Research Report for the Research Program on Institutional Support Systems for Sustainable Local Management of Irrigation in Water-Short Basins, IWMI, Irapuato, México.

Rodríguez, Claudia, Helena Cotler y Georgina Cairé (2003), "La descentralización de la gestión ambiental en México. El Caso de la Cuenca Lerma-Chapala”, Tercer Congreso Latinoamericano de Cuencas Hidrográficas, Arequipa, Perú.

Tortajada, Cecilia (2001), Environmental Sustainability of Water Management in Mexico, Third World Centre for Water Management, Mexico.

Tortajada, Cecilia (2002), “Abastecimiento de agua y manejo de descargas residuales en México: un análisis de las políticas ambientales”, en Patricia Ávila-García (ed.), Agua, 
cultura y sociedad en México, El Colegio de MichoacánInstituto Mexicano de Tecnología del Agua, Zamora, Michoacán.

UNESCO (2004), World Water Assessment Report, unESCO, París.

Vargas-Velázquez, Sergio (2003), "Política del agua y participación social: del modelo centralizado al modelo de gestión integral por cuenca", en Patricia Ávila-García (ed.), Agua, medio ambiente y desarrollo en el siglo XXI, El Colegio de Michoacán-Secretaría de Urbanismo y Medio AmbienteSemarnat-Instituto Mexicano de Tecnología del Agua, Zamora, Michoacán.

Vega-López, Obdulia y Héctor Raúl Pacheco-Vega (2000), “Zanahorias, palos y sermones: una revisión de la teoría de instrumentos de política ambiental", Revista Mexicana de Legislación Ambiental, 2 (4): 25-33.

Wester, Phillipus, Roberto Melville y Roberto Ramos-Osorio (2001), "Institutional Arrangements for Water Management in the Lerma-Chapala Basin", en A. Hansen y M. van Affereden (eds.), The Lerma-Chapala Watershed, Kluwer Academic Publishers-Plenum Publishers, Nueva York.

Wester, Phillipus, Douglas J. Merrey y Marna de Lange (2003), "Boundaries of Consent: Stakeholder Representation in River Basin Management in Mexico and South Africa”, World Development, 31 (5): 797-812.

Wester, Phillipus, Christopher A. Scott y Martin Burton (2005), "River Basin Closure and Institutional Change in Mexico's Lerma Chapala Basin”, en M. Svendsen (ed.), Irrigation and River Basin Management: Options for Governance and Institutions, САBI Publishers, Londres.

Recibido: 11 de agosto de 2005. Reenviado: 19 de octubre de 2005. Aprobado: 20 de octubre de 2006. 
Raúl Pacheco-Vega es actualmente investigador independiente; asociado por proyectos con el Centro de Innovación Aplicada en Tecnologías Competitivas (CIATEC). Al momento de escribir el artículo era investigador titular del Centro arriba señalado. Sus publicaciones más recientes son: "Ciudadanía ambiental global: un recorte analítico para el estudio de la sociedad civil transnacional", Espiral: Estudios sobre Sociedad y Estado, XII (35): 149171; "Democracy by Proxy: Environmental NGOs and Policy Change in Mexico", Aldemaro Romero y Sarah West (eds.), Environmental Issues in Latin America and the Caribbean, Springer Verlag, pp. 231-249; "E-gobierno en la difusión de información ambiental en Mexico", en Rodrigo Araya Dusijin y Miguel Ángel Porrúa-Vigón (eds.), América Latina Puntogov: casos y experiencias en Gobierno Electrónico, FLACSO-Chile, pp.189-208. 